\title{
Title: Global drivers of eukaryotic plankton biogeography in the sunlit ocean
}

Authors: Sommeria-Klein, Guilhem ${ }^{1 *}$; Watteaux, Romain ${ }^{2}$; Iudicone, Daniele ${ }^{2}$; Bowler,

$$
\text { Chris }^{1} \text {; Morlon, Hélène }{ }^{1 *}
$$

\section{Affiliations:}

$6 \quad{ }^{1}$ Ecole Normale Supérieure, PSL Research University, Institut de Biologie de l'Ecole Normale Supérieure (IBENS), CNRS 59 UMR 8197, INSERM U1024, 46 rue d’Ulm, F75005 Paris, France.

${ }^{2}$ Stazione Zoologica Anton Dohrn, Villa Comunale, 80121 Naples, Italy.

*Correspondence to: guilhem.sk@gmail.com; helene.morlon@bio.ens.psl.eu

Abstract (125/125 words): Eukaryotic plankton are a core component of marine ecosystems with exceptional taxonomic and ecological diversity. Yet how their ecology interacts with the environment to drive global distribution patterns is poorly understood. Here, we use Tara Oceans metabarcoding data covering all the major ocean basins combined with a probabilistic model of taxon co-occurrence to compare the biogeography of 70 major groups of eukaryotic plankton. We uncover two main axes of biogeographic variation. First, more diverse groups display stronger biogeographic structure. Second, large-bodied consumers are structured by oceanic basins, mostly via the main currents, while small-bodied phototrophs are structured by latitude, with a comparatively stronger influence of environmental conditions. Our study highlights striking differences in biogeographies across plankton groups and investigates their determinants at the global scale.

24 One-sentence summary (121/125 characters): Eukaryotic plankton biogeography and its

25 determinants at global scale reflect differences in ecology and body size. 
Main text: Marine plankton communities play key ecological roles at the base of oceanic

a key part of the effort to model the response of oceans to environmental changes (3-6). Part of the difficulty lies in the constant recirculation of plankton communities by ocean currents, along which many physical, chemical and biological processes - the so-called seascape (7) modify community composition (8). Recent planetary-scale ocean sampling expeditions have revealed that eukaryotic plankton are taxonomically and ecologically extremely diverse, possibly even more so than prokaryotic plankton (9). Eukaryotic plankton range from picosized $(0.2-2 \mu \mathrm{m})$ to meso-sized $(0.2-20 \mathrm{~mm})$ organisms and larger, thus covering an exceptional range of sizes. Eukaryotic plankton also cover a wide range of ecological roles, from phototrophs (e.g., Bacillariophyta, Haptophyta, Mamiellophyceae) to parasites (e.g., Marine Alveolates or MALVs), and from heterotrophic protists (e.g., Diplonemida, Ciliophora, Acantharea) to metazoans (e.g., Arthropoda and Chordata, respectively represented principally by Copepods and Tunicates). Understanding how these body size and ecological differences modulate the influence of oceanic currents and local environmental conditions on geographic distributions is needed if one wants to predict how eukaryotic communities, and therefore the trophic interactions and global biogeochemical cycles they participate in, will change with changing environmental conditions.

Previous studies suggested that all eukaryotes up to a size of approximately $1 \mathrm{~mm}$ are globally dispersed and primarily constrained by abiotic conditions (10). While this view has been revised, the influence of body size on biogeography is manifest $(11,12)$. In particular, a parallel study by Richter et al. (12), which quantified changes in plankton metagenomic composition and highlighted the underlying dynamics using transport time along main 
currents, found that the turnover is slower, rather than faster, with increasing body size. This suggests that, rather than influencing biogeography through its effect on abundance and ultimately dispersal capacity (i.e., larger organisms are more dispersal-limited; 10, 11), body size influences biogeography through its relationship with ecology and ultimately the sensitivity of communities to environmental conditions as they drift along currents. Under this scenario, the distribution of large long-lived generalist predators such as Copepods (Arthropoda) is expected to be stretched through large-scale transport by main currents ( 8 , 12-14), and yet to be patchy as a result of small-scale turbulent stirring (15). These contrasted views illustrate that little is known on how the interplay between body size, ecology, currents and the local environment shapes biogeography (16).

Here, we study plankton biogeography across all major eukaryotic groups in the sunlit ocean using $18 \mathrm{~S}$ rDNA metabarcoding data from the Tara Oceans global survey (including recently released data from the Arctic Ocean; 17). We also used transport times from Richter et al. (12), and the same environmental data. The data encompass 250,057 eukaryotic Operational Taxonomic Units (OTUs) sampled globally at the surface and at the Deep Chlorophyl Maximum (DCM) across 129 stations. We use a probabilistic model that allows identification of a number of 'assemblages', each of which represents a set of OTUs that tend to co-occur across samples $(18,19$; cf. Mat. \& Meth.). Each local planktonic community can then be seen as a sample drawn in various proportions from the assemblages. Across the Tara Oceans samples and considering all eukaryotic OTUs together, we identified 16 geographically structured assemblages, each composed of OTUs covering the full taxonomic range of eukaryotic plankton (Fig. 1, S1; Appendix). Local planktonic communities often cannot be assigned to a single assemblage, as would be typical for terrestrial macro-organisms on a fixed landscape $(20,21)$, but are instead mixtures of assemblages (Fig. 1A). This is consistent with previous findings suggesting that neighbouring plankton communities are 
continuously mixed and dispersed by currents $(8,12)$. Nevertheless, three assemblages are particularly represented and most communities are dominated by one of them (Fig. 1A). The most prevalent assemblage represents a set of OTUs (about one fifth of the total) that are globally ubiquitous except in the Arctic Ocean (assemblage 1, in dark red). This assemblage typically accounts for about half the number of OTUs in non-Arctic communities, and is particularly rich in parasitic groups such as MALV (Fig. 1B). The two others dominate, respectively, in the Arctic Ocean (assemblage 13, in cyan) and in the Southern Ocean

83 (assemblage 15, in marine blue), and are particularly rich in diatoms (Fig. 1B). Based on 84 similarity in their OTU composition, the assemblages cluster into three main categories corresponding to low, intermediate and high latitudes (Fig. 1B). The transition between communities composed of high-latitude and lower-latitude assemblages is fairly abrupt, and occurs around $45^{\circ}$ in the North Atlantic and $-47^{\circ}$ in the South Atlantic, namely at the latitude 1A\&B; 22). 

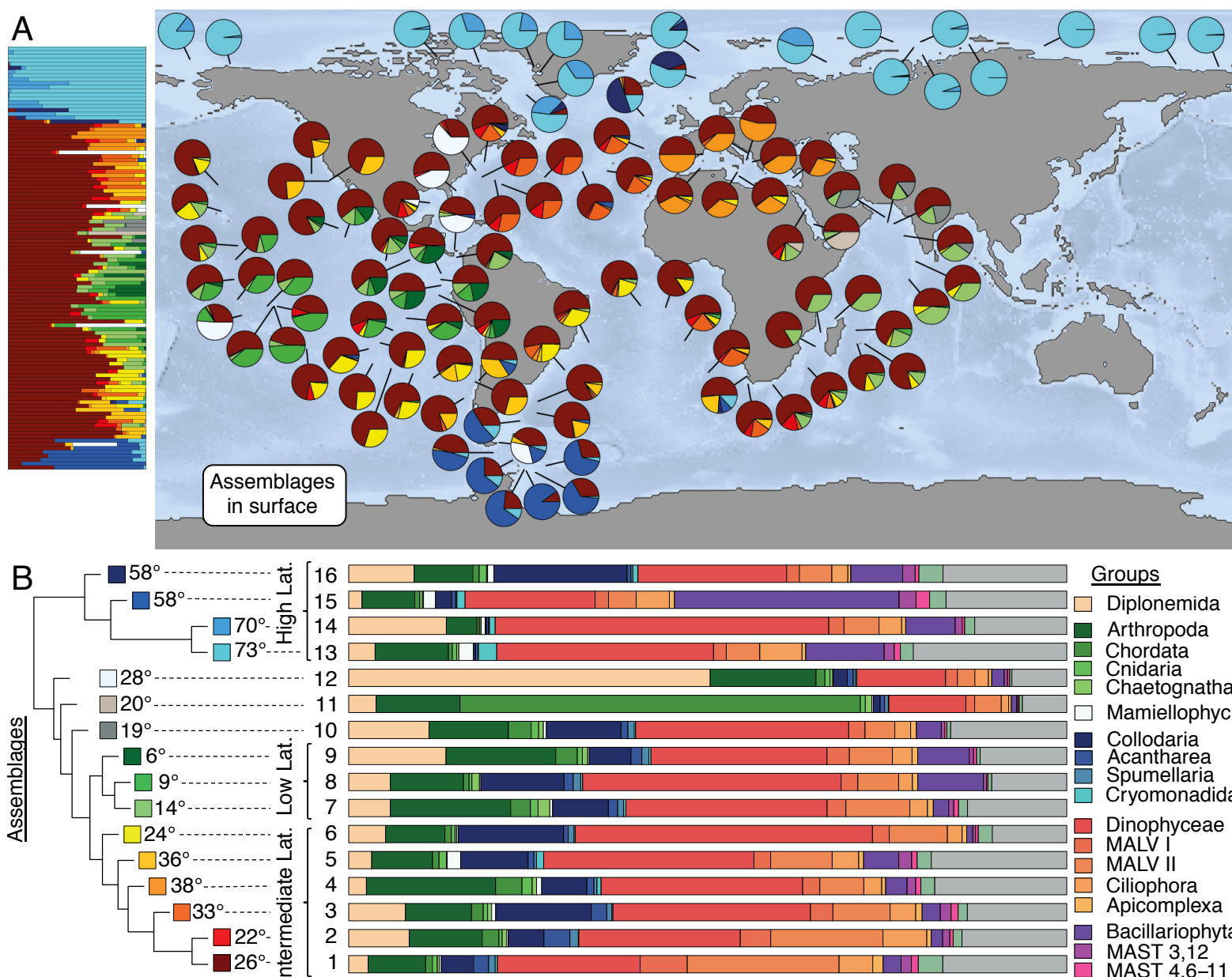

$\begin{array}{lllll}0.95 & 0.90 & 0.85 & 0.80 & 0.75\end{array}$

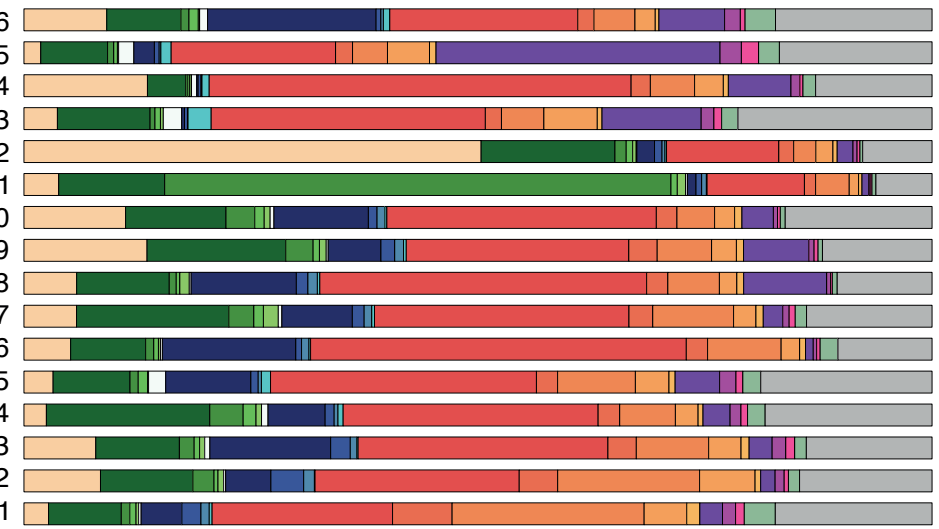

Group contribution to assemblage

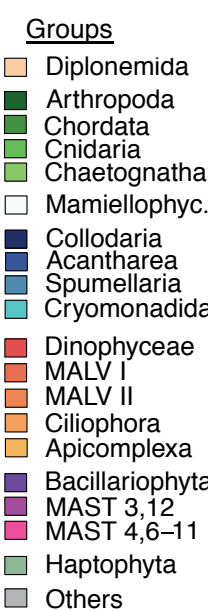

92 Fig. 1. Global surface biogeography of eukaryotic plankton. The biogeography of all eukaryotic

93 OTUs across Tara Oceans stations is characterized by 16 assemblages of co-occurring OTUs, each

94 represented by a distinct color (in A and left side of B) and identified by a number from 1 to 16 (in B).

95 (A) Relative contribution of the 16 assemblages to surface plankton community in Tara Oceans

96 stations, represented as pies on the world map, and as stacked bars vertically ordered by latitude on the

97 left-hand side of the map. (B) To the left: dendrogram of assemblage dissimilarity with respect to their

98 composition in OTUs (Simpson dissimilarity). The mean absolute latitude at which each assemblage is

99 found is indicated. Three clusters can be distinguished: a high-latitude cluster - the most distinctive

100 - in shades of blue, an intermediate-latidude cluster in shades from yellow to red, and a low-latitude

101 cluster in shades of green. To the right: barplot displaying the contribution of major eukaryotic groups

102 (deep-branching monophyletic groups) to assemblages. The 19 groups shown in the barplot are those

103 tallying more than 1,000 OTUs, grouped by phylogenetic relatedness. 
This global analysis hides a strong heterogeneity across the 70 most diversified deep-

Meth.), we found high pairwise dissimilarity values (ranging between 0.64 and 0.97; Fig. S2).

This heterogeneity can be decomposed into two main interpretable axes of variation (Fig. 2;

111 position on this axis is positively correlated to short-distance spatial autocorrelation

112 (Pearson's correlation coefficient $\rho=0.91$ at the surface; Fig. S3A), which measures the

113 tendency for close-by communities to be composed of the same assemblages (cf. Mat. \&

114 Meth.). Groups scoring low on this axis are characterized by strong local variation, or 115 "patchiness". The second axis reflects the nature of the biogeographic structure: group 116 position on this axis is positively correlated to the scale of biogeographic organization, which

117 we measured as the characteristic distance at which spatial autocorrelation vanishes $(\rho=$ $1180.53, p=10^{-6}$ at the surface; Fig. S3B) and which ranges from $\sim 7,000$ to $\sim 14,400 \mathrm{~km}$ across 119 groups. Group position on the second axis is also positively correlated to within-basin 120 autocorrelation ( $\rho=0.56, p=10^{-7}$ at the surface; Fig. S3C), which measures the tendency

121 for communities from the same oceanic basin (e.g., North Atlantic, South Atlantic, 122 Mediterranean, Southern Ocean) to be composed of the same assemblages, and negatively 123 correlated with latitudinal autocorrelation ( $\rho=-0.49, p=10^{-5}$ at the surface; S3D), which 124 measures the tendency for communities at the same latitude on both sides of the Equator to be 125 composed of the same assemblages (cf. Mat. \& Meth.). Results are similar at the DCM, 126 although less pronounced (Fig. S4). The 70 groups of eukaryotic plankton cover the full 127 spectra of biogeographies (Fig. 2, Fig. S5, Table S1), from those with weak spatial 128 organization, or high patchiness (i.e., scoring low on the first axis, such as Collodaria or 
bioRxiv preprint doi: https://doi.org/10.1101/2020 09.08 287524: this version posted December 24, 2020. The copyright holder for this preprint (which was not certified by peer review) is the author/funder, who has granted bioRxiv a license to display the preprint in perpetuity. It is made available under aCC-BY-NC-ND 4.0 International license.

129 Basidiomycota), to those organized at large spatial scale by oceanic basin (i.e., scoring high

130 on both axes, such as Chordata or Arthropoda), and those organized at smaller spatial scale

131 and according to latitude (i.e., scoring high on the first and low on the second axis, such as

132 Mamiellophyceae, Haptophyta or MAST 3,12). These striking differences across planktonic

133 groups suggest that accounting for their specificities is crucial to understanding their

134 biogeography.
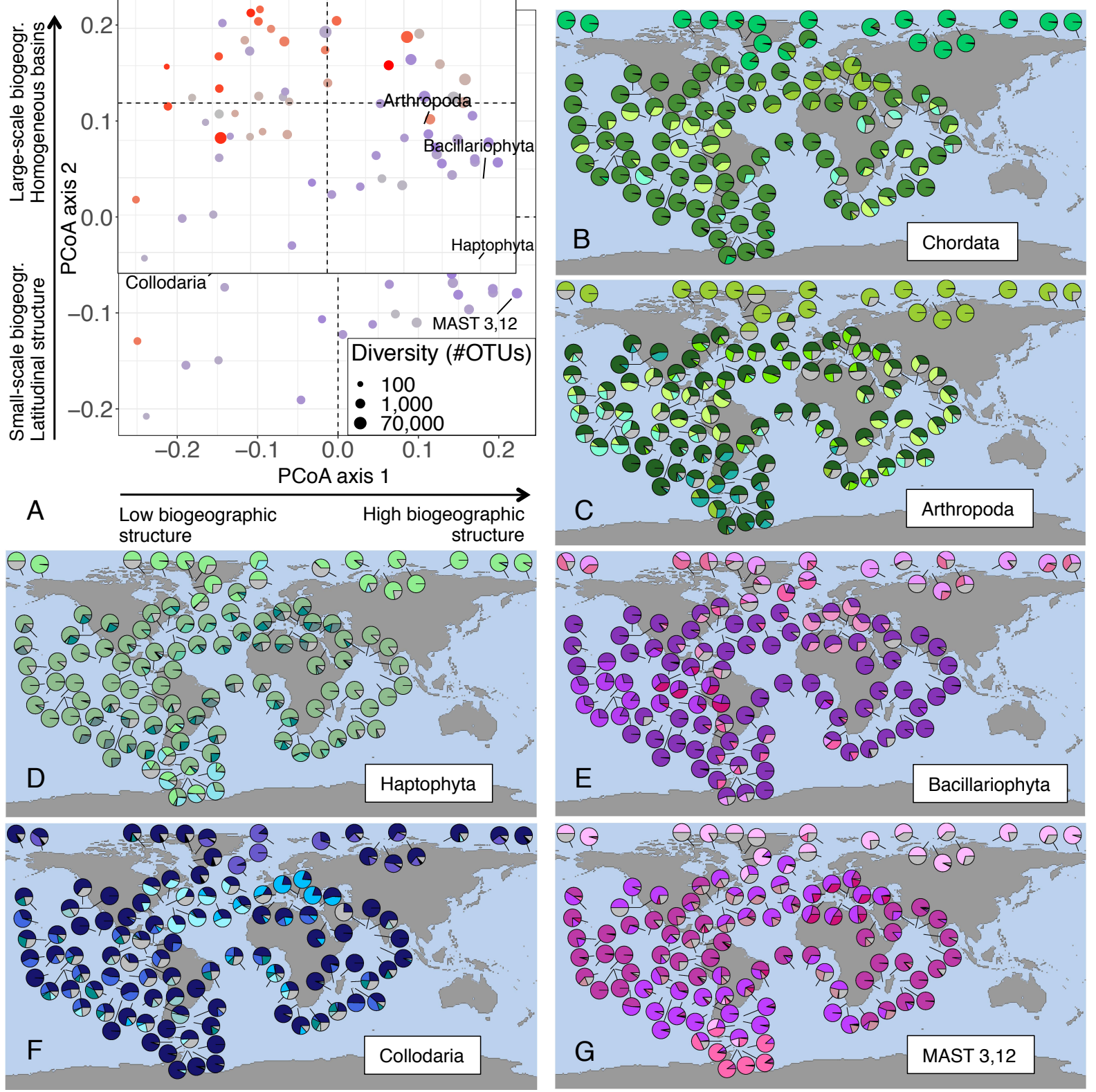

137 Fig. 2. Biogeographic heterogeneity across major eukaryotic plankton groups. (A) Principal

138 Coordinate Analysis (PCoA) of the biogeographic dissimilarity between 70 major groups of 
eukaryotic plankton. Each dot corresponds to the projection of a specific plankton group onto the first two axes of variation. Position along the first axis reflects the amount of biogeographic structure

141 displayed by the group, from a patchy distribution with weak short-distance spatial autocorrelation on

142 the left to a structured distribution with strong short-distance spatial autocorrelation on the right.

143 Position along the second axis reflects the nature of biogeographic structure, from a biogeography

144 structured by latitude at the bottom to a biogeography structured by oceanic basins at the top, as well

145 as the scale of biogeographic organization, from small to large scale. Dot size is proportional to the log 146 diversity of the corresponding group, and dot color represents its mean log body-size, from small 147 (blue) to large (red). (B-G) Surface biogeography of six major eukaryotic plankton groups. The 148 relative contribution of the 5 to 7 most prevalent assemblages is shown in color, and that of the 149 remaining assemblages is shown in gray; the color used for the most prevalent assemblage corresponds to the color used in Fig. 1B for the corresponding group. diversity, body size, and ecology, coarsely defined as either phototroph, phagotroph,

154 metazoan or parasite (cf. Mat. \& Meth.). We found that the amount of biogeographic structure 155 (group position on the first axis) is strongly correlated to diversity $\left(\rho=0.77, p=10^{-13}\right.$ 156 below 2,000 OTUs; Fig. 3A). This suggests that the maintenance of eukaryotic plankton 157 diversity over ecological and possibly evolutionary scales is tightly linked to biogeographic 158 structure, which may for example promote endemism. This relationship vanishes however for 159 groups larger than about 2,000 OTUs, and two of the most diverse groups (Diplonemida, 16038,769 OTUs and Collodaria, 17,417 OTUs) exhibit comparatively weak biogeographic 161 structure. The amount of biogeographic structure is weakly anticorrelated to body size $162(\rho=-0.32, p=0.007$; Fig. S6A), and after accounting for differences in diversity across 163 groups, is lower for metazoans than for phototrophs (ANCOVA t-test: $p=0.04$, Fig. S6B), in 164 agreement with the expectation of a higher local patchiness in their distribution induced by 
165 turbulent stirring $(15,24)$. In contrast, the nature of biogeographic structure (group position

166 on the second axis) is strongly correlated to body size $\left(\rho=0.64, p=10^{-9}\right.$; Fig. 3B) and

167 ecology (ANOVA F-test: $p=10^{-7}$, Fig. 3C), and only weakly to diversity $(\rho=0.24$,

$168 p=0.05$; Fig. S6C). Metazoan groups score high on the second axis of variation (with the

169 notable exception of Porifera sponges, probably at the larval stage, which are excluded from

170 statistical results) and phototrophs score low, while phagotrophs occupy an intermediate

171 position, spanning a comparatively wider range of biogeographies (Fig. 3C). Parasites are just

172 below metazoans, which suggests that their biogeography is influenced by that of their hosts.

173 While body size covaries with ecology (phagotrophs are larger than phototrophs on average,

174 and metazoans significantly larger than other plankton types; Fig. S7), the positive

175 relationship between group position on the second axis and body size still holds within each

176 of the four ecological categories (ANCOVA F-test: $p=10^{-4} ;$ Fig. S8). Diatoms

177 (Bacillariophyta) are a striking example: of all phototrophs, they have the largest body size

178 and also score highest on the second axis of variation. Conversely, ecology significantly

179 influences group position on the second axis even after accounting for body size differences

180 (ANCOVA F-test: $p=0.01$ ). Collodaria, which we did not assign to an ecological category,

181 score lower than expected from their large body size, but close to the average for

182 phagotrophic groups (Fig. 2, Table S1). These results suggest that biogeographic patterns are

183 influenced by both body size and ecology. To summarize, diversity-rich groups are

184 biogeographically structured, with large-bodied heterotrophs (metazoans such as Copepods

185 and Tunicates) exhibiting biogeographic variations at the scale of oceanic basins or larger,

186 and small-bodied phototrophs (such as Haptophyta) at smaller spatial scale and following

187 latitude (Fig. 2). 

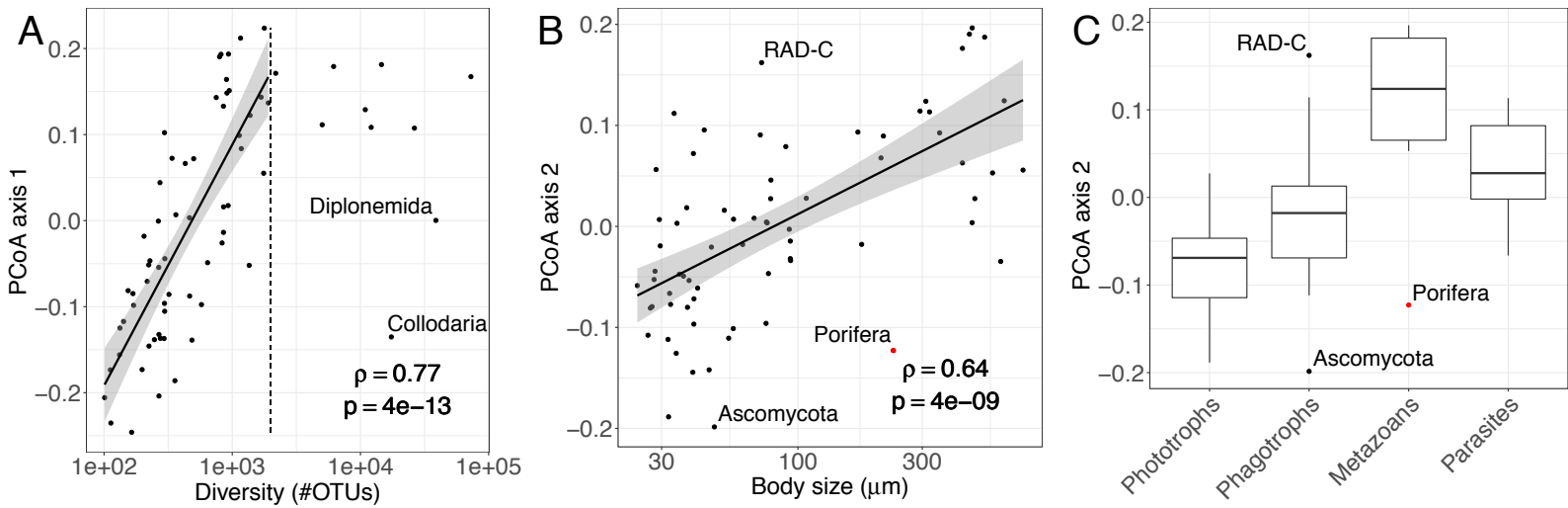

Fig. 3. Relationship between biogeography and diversity, mean body size and ecology across

major eukaryotic plankton groups. (A) The position of the 70 plankton groups along the first axis of

biogeographic variation, indicative of the amount of biogeographic structure, increases sharply with $\log$ diversity (number of OTUs in the group) up to approximately 2,000 OTUs, but not beyond (as exemplified by Diplonemida and Collodaria, two of the most diverse groups). (B) The position of the 70 plankton groups along the second axis, indicative of the nature and spatial scale of biogeographic

structure, increases with log mean body size, indicating that large-bodied plankton is organized at larger spatial scale and according to oceanic basins rather than latitude. (C) Positions along the second axis of plankton groups binned into four broad ecological categories (Collodaria and Dynophyceae were not categorized and are therefore not represented). Pairwise differences are all significant except between Phagotrophs and Parasites. The grey dot denotes Porifera, an outlier group excluded from statistical tests.

A global biogeography matching oceanic basins suggests that communities respond to environmental variations slowly enough to be homogenised by ocean circulation at the basin scale (i.e., gyres; 12), but have little ability to disperse between basins, either due to the comparatively limited connectivity by currents or to environmental barriers, and therefore that their biogeography is primarily shaped by the main ocean currents (13). Conversely, a 208 biogeography matching latitude, symmetric with respect to the Equator, suggests a faster response of communities to environmental variations within basins (which are structured by latitude and currents, e.g. the cross-latitudinal influence of the Gulf Stream), low cross-basin 
211 dispersal limitation, and therefore a comparatively more important role of local environmental

212 filtering in shaping biogeography. To explain the global biogeography of major taxonomic

213 groups, we compared biogeographic maps to maps of connectivity by currents and

214 environmental conditions. We transformed the matrix of minimum transport times between

215 pairs of stations, previously computed from a global ocean circulation model $(12,25)$, into

216 spatial patterns at different scales through eigenvector decomposition, thus obtaining a set of

217 so-called Moran Eigenvector maps (thereafter simply referred to as "connectivity maps"; cf.

218 Mat. \& Meth.). These maps represent the hypothetical geographic patterns expected for 219 plankton with temporal variation along currents matching these scales (Fig. S9, S10). We 220 estimated local abiotic conditions using yearly-averaged measurements of temperature, 221 nutrient concentration and oxygen availability (World Ocean Atlas 2013; 26; cf. Mat. \& 222 Meth.). Because biotic interactions (predation, competition, parasitic and mutualistic 223 symbiosis) are thought to be important determinants of plankton community structure (27), 224 we also quantified local biotic conditions using the relative read counts of major eukaryotic 225 groups (excluding the focal group; cf. Mat. \& Meth.). Biotic conditions, similarly to abiotic 226 ones, have a latitudinal structure, and we refer here to them collectively as 'environmental 227 conditions' (Fig. S11, S12). The resulting environmental maps can be interpreted as the 228 hypothetical geographic patterns expected for organisms with a fast response to local 229 environmental conditions and whose dispersal by currents is not limiting. Hence, a 230 biogeography matching connectivity maps better than environmental maps suggest that the 231 constraints imposed by the seascape, that is the transport of plankton by oceanic currents 232 modulated by mixing and ecological drift, but also by the responses to nutrient supplies and 233 temperature variations during transport, dominate over those imposed by detectable local 234 environmental filtering (see also 12). 
We found that the total variance in surface community composition that can be explained by connectivity maps and local environmental conditions (abiotic and biotic) averages $27 \%$ across groups (min. $0 \%$ for Porifera, max. $58 \%$ ) and is, as expected, tightly correlated to the amount of biogeographic structure ( $\rho=0.88$; Fig. 4A; cf. Mat. \& Meth.). The part of the variance that is statistically explained by connectivity patterns is primarily contributed by between-basin connectivity patterns (Fig. S10 \& S13), and is for most groups

241 larger than the part of the variance statistically explained by environmental data (at the surface, on average $40 \%$ of the explained variance is purely explained by connectivity versus $22 \%$ by the environment; Fig. S14A). This supports a prominent role of transport by the main current systems and of the processes occurring along those pathways in shaping eukaryotic plankton biogeography, both by extending the distribution of some taxa beyond their optimal range (28) and by constraining long-distance dispersal. Unmeasured environmental variations along currents likely contribute to this role of ocean circulation. As expected from our previous results, the ratio of the fractions of variance explained by connectivity patterns and environmental data, which reflects their relative contributions to biogeography, increases with group position on the second axis of variation $\left(\rho=0.44, p=10^{-4}\right.$; Fig. 4B). Accordingly, the relative contribution of connectivity by currents also increases with average group body size $\left(\rho=0.42, p=10^{-4}\right.$; Fig. 4C) and depends on ecology (ANOVA F-test: $p=0.003$; Fig. 4D). These results indicate that metazoans are closer to drifting tracers strongly influenced by 254 currents, and constrained in particular by limited between-basin connectivity, while 255 phototrophs are more strongly coupled with environmental factors and disperse more readily between basins. The difference in sensitivity to local environmental conditions can be 257 explained by differences in ecological requirements and community dynamics. Why there is a 258 difference in between-basins dispersal is less clear. All basins are connected by currents 259 within a few years of transport time (29), and small phototrophs may have a higher ability to 
260 disperse through environmental barriers by forming spores or dormant states (10).

261 Alternatively, the looser environmental coupling and slower dynamics of metazoan

262 communities might make them more sensitive to the smaller between-basin compared to

263 within-basin water flow. Finally, within the variance explained by the local environment, an

264 approximately equal share can be attributed to biotic and abiotic conditions for most groups

265 (respectively $29 \%$ and 26\% purely explained at the surface, on average; Fig. S14B),

266 irrespective of their body size, ecology, diversity or biogeography (Fig. S15). Results are

267 similar at the DCM, but are far less pronounced (Fig. S16, S17). Although we cannot exclude

268 the possibility that local biotic conditions reflect the indirect effect of local abiotic factors that

269 are not accounted for in our study, such as fluxes of nutrients, which are often more relevant

270 to planktonic organisms than instantaneous nutrient concentrations (28), these results indicate

271 an additional role for interspecific interactions in shaping community composition $(27,30)$.
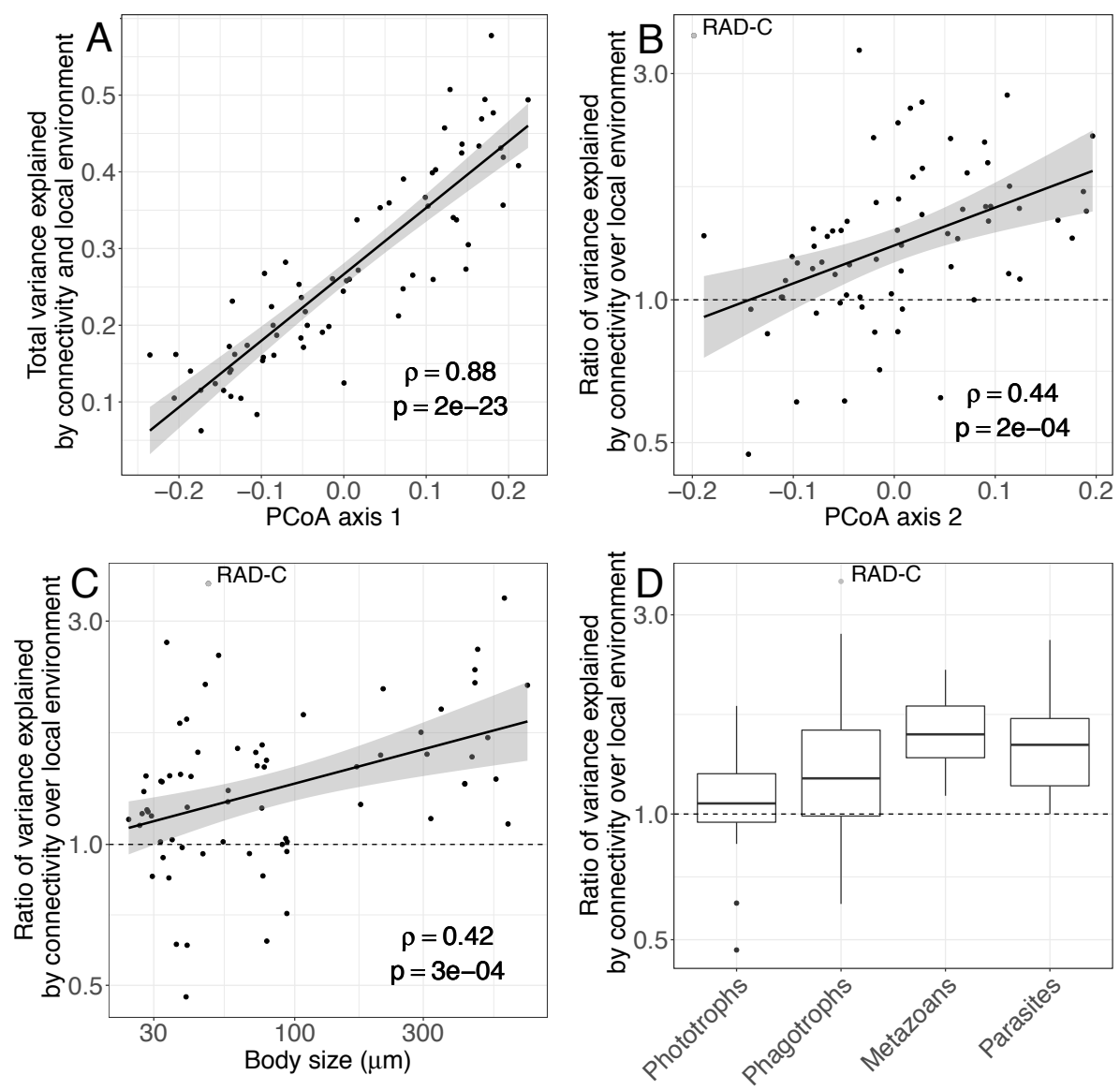
274 Fig. 4. Drivers of surface biogeography across major eukaryotic plankton groups. (A) The total

275 variance in surface biogeography that can be explained by the combination of connectivity by currents

276 and (abiotic and biotic) local environmental conditions increases with the position of plankton groups

277 on the first axis of biogeographic variation. (B-D) Across major plankton groups, the log ratio of the 278 variance explained by connectivity over the variance explained by (abiotic and biotic) local 279 environmental conditions (B) increases with group position on the second axis of variation, (C) 280 increases with $\log$ mean body size, and (D) varies across broad ecological categories (pairwise 281 differences are significant except Metazoans-Parasites and Phagotrophs-Phototrophs). The ratio is 282 higher than 1 for most groups, reflecting an overall stronger influence of connectivity by currents 283 compared to local environmental conditions on plankton biogeography at the surface. The grey dot 284 denotes RAD-C, an outlier group excluded from statistical tests. We did not find any significant explanatory variable for Porifera and therefore excluded this group from these analyses. the main groups of eukaryotic plankton in the sunlit ocean. Consistent with metagenomic results at lower taxonomic resolution (12), we find that eukaryotic plankton exhibits a globalscale biogeography, and that community variation is slow enough along currents to allow them to be the dominant driver of this biogeography. The continuous movement of water masses generates biogeographic patterns that are better represented by overlapping taxa assemblages than by the well-delineated biomes characteristic of terrestrial systems. Our 294 comparison of eukaryotic plankton groups reveals several additional results. First, the 295 geographic structuring induced by currents may have favored the generation and maintenance 296 of eukaryotic plankton diversity. Second, plankton ecology matters beyond body size 297 differences, and reciprocally body size matters beyond ecological differences. Third, body 298 size and ecology influence primarily the nature of biogeographic patterns, namely their spatial 299 scale of organization and whether they are organized by oceanic basins or latitude, and only 
300 secondarily the amount of biogeographic structure, namely local patchiness. Fourth, biotic

301 conditions appear to be at least as important a driver of biogeography as local abiotic

302 conditions. Our results reconcile the views that larger-bodied organisms are more dispersal-

303 limited $(10,11)$ and yet display a slower compositional turnover along currents than smaller

304 organisms (12): at the global scale, organisms of larger sizes are indeed more dispersal-

305 limited; however at the regional scale, they have wider spatial distributions, presumably

306 linked to their specific ecologies, longer lifespan and reduced sensitivity to local

307 environmental variations. At the two extremes, metazoan heterotrophs are structured at the

308 scale of oceanic basins following the main currents, while small phototrophs are structured

309 latitudinally with a comparatively larger influence of local environmental conditions,

310 including biotic ones. Together, our results suggest that predictive modeling of plankton

311 communities in a changing environment $(17,31)$ will critically depend on our ability to model

312 the impact of changes in ocean currents and to develop niche models accounting for both

313 species ecology and interspecific interactions. 


\section{References and Notes}

315

316 1. C. B. Field, M. J. Behrenfeld, J. T. Randerson, P. Falkowski, Primary production of the biosphere: integrating terrestrial and oceanic components. Science. 281, 237-240 (1998).

2. A. Z. Worden, M. J. Follows, S. J. Giovannoni, S. Wilken, A. E. Zimmerman, P. J. Keeling, Rethinking the marine carbon cycle: Factoring in the multifarious lifestyles of microbes. Science. 347, 1257594 (2015).

3. G. Beaugrand, R. R. Kirby, How Do Marine Pelagic Species Respond to Climate Change? Theories and Observations. Annual Review of Marine Science. 10, 169-197 (2018).

4. E. J. Raes, L. Bodrossy, J. van de Kamp, A. Bissett, M. Ostrowski, M. V. Brown, S. L. of Sciences. 115, E8266-E8275 (2018). phytoplankton diversity driven by temperature and environmental variability. Science Advances. 5, eaau6253 (2019). Global patterns and predictors of marine biodiversity across taxa. Nature. 466, 10981101 (2010).

7. M. T. Kavanaugh, M. J. Oliver, F. P. Chavez, R. M. Letelier, F. E. Muller-Karger, S. C. Doney, Seascapes as a new vernacular for pelagic ocean monitoring, management and conservation. ICES J Mar Sci. 73, 1839-1850 (2016). 
community structure affected by oceanic dispersal and mesoscale turbulence. Limnol.

9. C. de Vargas, S. Audic, N. Henry, J. Decelle, F. Mahe, R. Logares, E. Lara, C. Eukaryotic plankton diversity in the sunlit ocean. Science. 348, 1261605 (2015).

10. B. J. Finlay, Global Dispersal of Free-Living Microbial Eukaryote Species. Science.

11. E. Villarino, J. R. Watson, B. Jönsson, J. M. Gasol, G. Salazar, S. G. Acinas, M.

12. D. J. Richter, R. Watteaux, T. Vannier, J. Leconte, P. Frémont, G. Reygondeau, N. Maillet, N. Henry, G. Benoit, A. Fernàndez-Guerra, S. Suweis, R. Narci, C. Berney, D. Eveillard, F. Gavory, L. Guidi, K. Labadie, E. Mahieu, J. Poulain, S. Romac, S. Roux, C. Dimier, S. Kandels, M. Picheral, S. Searson, T. O. Coordinators, S. Pesant, J.-M. Aury, J. R. Brum, C. Lemaitre, E. Pelletier, P. Bork, S. Sunagawa, L. KarpBoss, C. Bowler, M. B. Sullivan, E. Karsenti, M. Mariadassou, I. Probert, P. 
Peterlongo, P. Wincker, C. de Vargas, M. R. d'Alcalà, D. Iudicone, O. Jaillon, T. O. Coordinators, Genomic evidence for global ocean plankton biogeography shaped by large-scale current systems. bioRxiv, 867739 (2019).

13. F. L. Hellweger, E. van Sebille, N. D. Fredrick, Biogeographic patterns in ocean microbes emerge in a neutral agent-based model. Science. 345, 1346-1349 (2014).

14. M.-A. Madoui, J. Poulain, K. Sugier, M. Wessner, B. Noel, L. Berline, K. Labadie, A. Cornils, L. Blanco-Bercial, L. Stemmann, J.-L. Jamet, P. Wincker, New insights into global biogeography, population structure and natural selection from the genome of the epipelagic copepod Oithona. Molecular Ecology. 26, 4467-4482 (2017).

15. E. R. Abraham, The generation of plankton patchiness by turbulent stirring. Nature. 391, 577-580 (1998).

16. L. Oziel, A. Baudena, M. Ardyna, P. Massicotte, A. Randelhoff, J.-B. Sallée, R. B. temperate phytoplankton in the Arctic Ocean. Nat Commun. 11, 1-8 (2020). Coelho, H. Endo, J. M. Gasol, A. C. Gregory, F. Mahé, J. Rigonato, M. Royo-Llonch, 
Trends in Marine Plankton Diversity across Kingdoms of Life. Cell. 179, 10841097.e21 (2019).

18. G. Sommeria-Klein, L. Zinger, E. Coissac, A. Iribar, H. Schimann, P. Taberlet, J. Chave, Latent Dirichlet Allocation reveals spatial and taxonomic structure in a DNAbased census of soil biodiversity from a tropical forest. Molecular Ecology Resources. 20, 371-386 (2019).

19. D. Valle, B. Baiser, C. W. Woodall, R. Chazdon, Decomposing biodiversity data using the Latent Dirichlet Allocation model, a probabilistic multivariate statistical method. Ecology Letters. 17, 1591-1601 (2014).

20. A. R. Wallace, The geographical distribution of animals: with a study of the relations of living and extinct faunas as elucidating the past changes of the earth's surface (Cambridge University Press, 1876), vol. 1.

21. G. F. Ficetola, F. Mazel, W. Thuiller, Global determinants of zoogeographical boundaries. Nature Ecology and Evolution. 1, 1-7 (2017).

22. L. D. Talley, Descriptive physical oceanography: an introduction (Academic press, 2011).

23. M. Meila, Comparing clusterings — an information based distance. Journal of Multivariate Analysis. 98, 873-895 (2006). from zooplankton to seabirds. Nat Commun. 5, 1-9 (2014). Biogeochemical versus ecological consequences of modeled ocean physics.

413 26. T. P. Boyer, J. I. Antonov, O. K. Baranova, C. Coleman, H. E. Garcia, A. Grodsky, D. 

2013. (2013), doi:10.7289/V5NZ85MT.

27. G. Lima-Mendez, K. Faust, N. Henry, J. Decelle, S. Colin, F. Carcillo, S. Chaffron, J. of community structure in the global plankton interactome. Science. 348, 1262073

28. S. Dutkiewicz, P. Cermeno, O. Jahn, M. J. Follows, A. E. Hickman, D. A. A.

29. B. F. Jönsson, J. R. Watson, The timescales of global surface-ocean connectivity. Nature Communications. 7, 11239 (2016).

30. F. Vincent, C. Bowler, Diatoms Are Selective Segregators in Global Ocean Planktonic Communities. mSystems. 5 (2020), doi:10.1128/mSystems.00444-19.

31. H. K. Lotze, D. P. Tittensor, A. Bryndum-Buchholz, T. D. Eddy, W. W. L. Cheung, E. D. Galbraith, M. Barange, N. Barrier, D. Bianchi, J. L. Blanchard, L. Bopp, M. 
projections reveal trophic amplification of ocean biomass declines with climate change. Proc. Natl. Acad. Sci. U.S.A. 116, 12907-12912 (2019).

32. F. Mahé, T. Rognes, C. Quince, C. de Vargas, M. Dunthorn, Swarm: robust and fast clustering method for amplicon-based studies. PeerJ. 2, e593 (2014). (2013).

34. D. M. Blei, A. Y. Ng, M. I. Jordan, Latent Dirichlet Allocation. Journal of Machine Learning Research. 3, 993-1022 (2003).

35. X.-H. Phan, L.-M. Nguyen, S. Horiguchi, in Proceeding of the 17th international conference on World Wide Web - WWW '08 (ACM Press, Beijing, China, 2008;

36. B. Grün, K. Hornik, topicmodels: an R package for fitting topic models. Journal of statistical software. 40, 1-30 (2011).

37. R Core Team, $R$ : A Language and Environment for Statistical Computing (R Foundation for Statistical Computing, Vienna, Austria, 2018).

460 38. E. Paradis, K. Schliep, ape 5.0: an environment for modern phylogenetics and evolutionary analyses in R. Bioinformatics. 35, 526-528 (2019).

462 39. P. Legendre, L. Legendre, Numerical Ecology (Elsevier, 2012).

463 40. D. Menemenlis, J.-M. Campin, P. Heimbach, C. Hill, T. Lee, M. Schodlok, H. Zhang, 
41. D. Chessel, A. Dufour, J. Thioulouse, The ade4 Package - I: One-Table Methods. $R$ News, 5-10 (2004).

42. J. R. Watson, C. G. Hays, P. T. Raimondi, S. Mitarai, C. Dong, J. C. McWilliams, C. community similarity and ocean circulation. Ecology. 92, 1193-1200 (2011).

43. D. Wilkins, E. van Sebille, S. R. Rintoul, F. M. Lauro, R. Cavicchioli, Advection environment effects. Nature Communications. 4, 1-7 (2013).

44. J. Oksanen, F. G. Blanchet, M. Friendly, R. Kindt, P. Legendre, D. McGlinn, P. R. Minchin, R. B. O’Hara, G. L. Simpson, P. Solymos, M. H. H. Stevens, E. Szoecs, H. Wagner, vegan: Community Ecology Package (2018).

45. Clayton Sophie, Dutkiewicz Stephanie, Jahn Oliver, Follows Michael J., Dispersal, eddies, and the diversity of marine phytoplankton. Limnology and Oceanography: Fluids and Environments. 3, 182-197 (2012).

480 46. S. Dutkiewicz, P. Cermeno, O. Jahn, M. J. Follows, A. E. Hickman, D. A. A. Taniguchi, B. A. Ward, Dimensions of Marine Phytoplankton Diversity. Biogeosciences Discussions, 1-46 (2019).

483 47. J. Hausser, K. Strimmer, entropy: estimation of entropy, mutual information and related quantities (2014). 


\section{Acknowledgements}

We are grateful to Federico Ibarbalz for his essential help with the data. We thank Olivier

Jaillon and Colomban de Vargas for feedback and early discusions on the project. We thank

Mick Follows and Oliver Jahn for sharing MITgem simulation results for the Arctic Ocean.

491 We thank Florian Hartig and Odile Maliet for their guidance on Bayesian inference, Leandro

Arístide and Felipe Delestro for their kind assistance with the figures, and Carmelo Fruciano

Kazamia, Sophia Lambert, Eric Lewitus, Marc Manceau, Olivier Missa, Silvia de Monte,

Isaac Overcast, Ignacio Quintero, Enrico Ser-Giacomi, Ana Catarina Silva and Flora Vincent

496 for suggestions and fruitful discussions.

Funding: This work was supported by European Research Council grants (ERC 616419-

PANDA, to H.M.; ERC 835067-DIATOMIC, to C.B.), grants from the French Agence

Nationale de la Recherche (MEMOLIFE, ref. ANR-10-LABX-54, to G.S.K., H.M. and C.B.;

501 OCEANOMICS, ref. ANR-11-BTBR-0008, to C.B.) and funds from CNRS. C.B. and H.M.

Oceans.

506 Author contributions: GSK and HM designed the study with the help of RW, DI and CB.

507 GSK performed the analyses. RW contributed the transport time data and their interpretation.

510 Competing interests: The authors declare no competing financial interests. 
512 Data availability: All data reported herein are available without restrictions. Metabarcoding

513 data have been deposited at the European Nucleotide Archive (ENA) under accession

514 numbers PRJEB6610 and PRJEB9737. Sample metadata are available from

515 https://doi.org/10.1594/PANGAEA.875582. 


\section{List of Supplementary Material}

517

518 Materials and Methods

519 Appendix

520 Figures S1 to S17

521 Table S1

522 References (32-47) 